\title{
COMMUNICATION
}

\section{Amylose AL linguale compliquant un myélome multiple : à propos d'un cas}

\author{
Cambronne C, Catros S, Fenelon M, Fricain J-C
}

Pôle d'odontologie et de santé buccale, CHU de Bordeaux

Introduction

L'amylose est une pathologie caractérisée par le dépôt et l'accumulation extracellulaire d'une substance protéique dite " amyloïde ». Dans l'amylose AL (amylose " primitive »), les fibrilles de la substance amyloïde sont constituées par la polymérisation de fragments monoclonaux de chaînes légères d'immunoglobulines. L'amylose peut toucher un ou plusieurs organes, dont elle perturbe l'architecture et la fonction. L'atteinte linguale a peu été décrite dans la littérature. Un nouveau cas sévère d'amylose AL linguale est rapporté.

\section{Observation}

Un patient de 62 ans, était adressé pour une gêne linguale fonctionnelle importante associée à des douleurs. Il était atteint d'un myélome multiple depuis 2006, traité initialement par melphalan et autogreffe, puis par plusieurs lignes de chimiothérapie en fonction des épisodes évolutifs. Une amylose AL avait été évoquée en 2014 suite à la survenue d'un syndrome du canal carpien bilatéral. Lors de son traitement chirurgical, une biopsie synoviale avait révélé la présence de dépôts amyloïdes. À l'examen clinique on retrouvait une macroglossie avec indentations latérales. La langue était indurée, ankylosée, et douloureuse à la palpation. Le patient rapportait une grande difficulté à s'alimenter (dysphagie, odynophagie, incapacité à déglutir les aliments solides) à l'origine d'une altération de l'état général avec amaigrissement. La phonation était altérée, et l'hygiène orale compromise. La biopsie et l'examen anatomo-pathologique confirmèrent la localisation linguale de lésion d'amylose. Un ajustement occlusal au niveau des dents cuspidées ainsi qu'une infiltration locale de Triamcinolone furent réalisés, mais aucune amélioration clinique ne fut obtenue.

\section{Discussion}

Le myélome multiple, caractérisé par une prolifération plasmocytaire maligne, se manifeste par la synthèse massive d'une paraprotéine monoclonale immunoglobulinique. Lorsqu'il s'agit d'un fragment de chaîne légère, il peut se compliquer d'une amylose AL. Les dépôts amyloïdes au niveau de la langue sont alors fréquemment à l'origine de macroglossies plus ou moins invalidantes. La macroglossie peut même être le premier signe clinique révélateur d'une amylose primaire, voire d'un myélome (Christiaens et al, 1999). Le principe du traitement de l'amylose vise à réduire la charge protéique en agissant sur la prolifération monoclonale sous-jacente. En raison d'un manque de données et de l'absence de preuve scientifique, il n'existe pas de consensus quant à la gestion de la macroglossie due à une amylose systémique. Le traitement chirurgical 
par glossectomie partielle peut être envisagé en cas d'impotence fonctionnelle ou esthétique majeure, dans la mesure ou la prolifération monoclonale sous-jacente est supprimée (Pau et al., 2012). Dans le cas présent, l'importance de la gêne fonctionnelle consécutive à la macroglossie nécessitait une prise en charge symptomatique. Le risque de récidive, l'imprédictibilité du résultat postopératoire et l'importante morbidité inhérente à la glossectomie (Guijarro-Martinez et al., 2009) font que ce traitement n'a pas été retenu en première intention. 